\title{
Personalized medicine in advanced urothelial cancer: when to treat, how to treat and who to treat
}

\author{
Behfar Ehdaie, MD; ${ }^{*}$ Steven C. Smith, MD, PhD; ${ }^{\dagger}$ Dan Theodorescu, MD, PhD ${ }^{* \neq \neq}$
}

\begin{abstract}
The past decade has provided an improved understanding of the molecular mechanism of bladder cancer by defining distinct pathways in tumorigenesis and progression. Advances in technologies, such as high-throughput transcript profiling, microarrays and proteomics, offer a systematic approach to identifying targets for bladder cancer diagnostics and drug discovery. This review presents a select outline of the advances in the development of biomarkers and targets for patient prognosis and therapy selection. This paper describes a representative cohort of recent studies that have the potential to significantly impact the management of muscle invasive and metastatic urothelial carcinoma of the bladder. Space constraints do not permit this review to be comprehensive and we apologize to the authors whose work we do not cite.
\end{abstract}

Can Urol Assoc J 2009;3(Supp14):S232-6

\section{Introduction}

Bladder cancer is a common malignant disease, with 357000 new cases and 145000 deaths worldwide per year. ${ }^{1}$ It is the fifth most common neoplasm in industrialized countries and the second most common genitourinary malignancy in the United States. ${ }^{2}$ Urothelial carcinomas (UC), formerly known as transitional cell carcinomas, comprise $90 \%$ of all carcinomas of the bladder in Western countries; histology findings identify $5 \%$ as squamous and $2 \%$ as adenocarcinoma. Urothelial carcinoma is the focus of this review.

Molecular and histopathologic studies indicate that urothelial carcinomas present as a heterogeneous group of tumours that may evolve along dual pathways with distinct biological behaviours and clinical prognosis. ${ }^{3,4}$ In most cases, UC presents as papillary or non-muscle-invasive (clinical stage $\mathrm{Ta}, \mathrm{T} 1)$. The natural history of these tumours significantly affects local recurrence rates and infrequent progression to muscle invasion or metastases. ${ }^{5}$ In contrast, muscle-invasive UC (clinical stage $\geq T 2$ ) is a lethal malignancy that, when untreated, results in death within 2 years of the diagnosis in over $85 \%$ of patients. ${ }^{6}$ A definitive surgical approach that involves removing the primary bladder tumour and regional lymph nodes results in excellent long-term survival rates. ${ }^{7}$ However, despite improved outcomes with neoadjuvant cisplatin-based combined chemotherapy, almost half of these patients will relapse with metastatic disease. ${ }^{8}$ Conventional cisplatin-based chemotherapy regimens for advanced disease include methotrexate, vinblastine, doxorubicin and cisplatin (MVAC); dose-dense MVAC; and gemcitabine/ cisplatin (GC). Despite initial high response rates, overall 5 -year survival is suboptimal at 5\% to $20 \%$. $^{9-11}$

Several studies have evaluated the clinical and pathological prognostic factors after cystectomy for muscle-invasive UC. Advanced pathologic stage, nodal involvement, tumour size greater than $3 \mathrm{~cm}$, elevated creatinine and lymphovascular invasion are independent risk factors for recurrence, ${ }^{12-15}$ while advanced pathologic stage and nodal involvement are independent prognostic factors for survival. ${ }^{14,16}$ A nomogram predicting recurrence risk after radical cystectomy for bladder cancer was recently developed to improve the predictability of accurate risk assessment in patients after this procedure. ${ }^{17}$ While these traditional prognostic factors provided useful estimates for recurrence risk and survival, significant variations within each prognostic group based on the heterogeneity of tumour biology were observed

Similarly, patients with locally advanced (T4b and N2-3) or metastatic disease (M1) at diagnosis or during follow-up demonstrate variable response rates to chemotherapy. Currently, Karnofsky Performance scores and presence of visceral metastases are reported to correlate with outcome of treatment. ${ }^{18}$ Given the molecular knowledge of urothelial tumorigenesis and chemosensitivity, more precise methods for predicting response to anticancer therapy seem possible.

The past decade has seen an exponential accumulation of research on molecular markers in bladder cancer. Biomarkers that enhance the predictive ability of standard clinicopathologic information and optimize prognostication are being discovered. ${ }^{19-30}$ In addition, advanced technologies offer a systematic approach for identifying active targets for drug discovery and tailored therapeutics in bladder cancer. The method described here defines a "personalized selection" approach to advanced bladder cancer within this increasingly tailored diagnostic and therapeutic framework, since optimizing management of a patient's 
disease is based on specific characteristics. These factors not only include traditional ones, such as age, gender, race, environment and tumour-specific clinicopathologic parameters, but also increasingly incorporate molecular profiling of genetic, genomic or proteomic factors of patient or tumour that drive or are at least are associated with prognosis and treatment response.

This review explores recent advances laying the groundwork toward making "personalized selection" a reality for patients with muscle invasive and metastatic bladder cancer. Equally important is the stratification of patients with non-muscle-invasive disease into risk groups for progression and treatment response; this will not be discussed in detail here.

\section{Recent developments}

Clinical decision-making has evolved from physician judgment and prognostic risk group stratification to prediction models using Cox multivariate regression and nomograms that attempt individualized prediction of outcomes. Additionally, prediction models based on the American Joint Committee on Cancer stage groupings have expanded to include histopathologic criteria and molecular expression signatures. Protein expression profiling of $U C$ has resulted in the identification of immunohistochemical markers that offer an alternative method to distinguish aggressive tumour biology and improve prognostic models in patients with advanced bladder UC treated with radical cystectomy. Concurrently, advanced technologies are enabling systematic discovery of novel biomarkers from genome-wide candidates.

\section{Biomarkers of prognosis and therapeutic efficacy}

The p53 tumour suppressor protein is a key gatekeeper in cell cycle control and plays an integral role in genome stability. ${ }^{31}$ Overexpression of p53, as determined by immunohistochemistry, is used to measure TP53 gene mutations. Many studies support the association of p53 nuclear accumulation with tumour stage, lymphovascular invasion, lymph node metastasis, high-grade tumour, disease recurrence and bladder cancer specific death. ${ }^{19-25}$ However, a metaanalysis by Malats that reviewed 117 studies spanning 10 years concluded that there is insufficient evidence to support that changes in p53 can be a marker of outcome in patients with bladder cancer. ${ }^{32}$ A clinical trial investigated whether alterations of immunohistochemical p53 nuclear expression could prospectively identify patients that would benefit from the administration of adjuvant MVAC chemotherapy. ${ }^{33}$ While results are not yet published, this study was the first to use a biomarker for personalized therapy assignment.

P21 and p27 proteins are cyclin-dependent kinase inhibitors and downstream targets of $\mathrm{p} 53$. In retrospective studies using tumour samples from radical cystectomy specimens, nuclear accumulation of p21 was detected in $64 \%$ of specimens and was an independent predictor of tumour recurrence and survival when concomitantly assessed with tumour grade, stage, lymph node status and p53 status. ${ }^{22,26}$ P27 overexpression in human cells has been shown to result in cell cycle arrest in the G1 phase. In patients with muscleinvasive disease treated with radical cystectomy, p27 was the second most powerful cell cycle regulator after p53 for prediction of bladder cancer recurrence and survival. ${ }^{27,28}$

Apoptosis (programmed cell death) results from a cascade of intrinsic and extrinsic signals and depends on the balance between pro- and anti-apoptotic proteins. ${ }^{34}$ Caspase-3 is a pro-apoptotic enzyme that has been associated with higher pathologic grade, stage and presence of lymph node metastasis in radical cystectomy specimens. ${ }^{29}$ In a study using multiple apoptosis markers to assess their value in improving the accuracy of predicting outcomes for patients with UC, Karam and colleagues demonstrated that loss of caspase-3 was an independent predictor of bladder cancerspecific survival after radical cystectomy. ${ }^{29} \mathrm{Bcl}-2$ is an antiapoptotic protein present in upstream events in apoptosis activation. Overexpression of $\mathrm{Bcl}-2$ is correlated with higher pathologic stage, disease recurrence and cancer-specific mortality rates, ${ }^{29}$ as well as lower response rates to chemotherapy. ${ }^{35}$ Survivin, an anti-apoptotic molecule that blocks downstream caspase activity, may be detected in urine at the protein or mRNA level and is associated with cancer presence, higher tumour grade and advanced pathologic stage. ${ }^{36}$ In radical cystectomy tumour samples, the proportion of specimens with survivin overexpression increased with tumour stage progression and correlated with diseasespecific mortality. ${ }^{37}$

The fact that so many biomarkers have been associated with key clinicopathologic characteristics suggests that prediction of prognosis based on a panel of genes might be more accurate than the evaluation of a single marker. Karam and colleagues found that p53, Bcl-2, caspase- 3 and survivin provided distinct correlations with tumour stage, grade and lymphovascular invasion. The total number of altered markers was a significant prognostic factor in disease recurrence and disease-specific survival. ${ }^{29}$ Similarly, Shariat and colleagues demonstrated that the addition of altered cell cycle biomarkers increased the predictive accuracy of nomogram-based disease recurrence and disease-specific survival by $10 \% .{ }^{30}$ Finally, improved characterization of the molecular pathways driving bladder cancer resulted in the identification of disease biomarkers that suggest the following therapeutic angles: mutations of fibroblast growth factor receptor 3 tyrosine kinase receptor are associated with superficial bladder cancer and a promising target for novel therapies. ${ }^{38}$ The epidermal growth factor receptor (EGFR) pathway, associated with invasion and poor prognosis, may 
be targeted with EGFR-family tyrosine kinase inhibitors. ${ }^{39}$ In our group, we have identified two novel, independent prognostic factors for patient prognosis that themselves suggest new therapeutic angles. The first factor is the association of loss of expression of the RhoGDI2 metastasis suppressor protein with decreased patient survival; ${ }^{40}$ this factor resulted in a preclinical evaluation of antagonists of endothelin receptor signaling through the discovery of that RhoGDI2 regulates this pathway. ${ }^{41}$ The second factor is our observation that increased CD24 expression independently predicts poor patient survival; ${ }^{42}$ this resulted in a preclinical evaluation of monoclonal antibodies for targeting CD24 positive cancer cells in vivo. ${ }^{43}$

\section{Genome-wide discovery of multiplexed biomarkers}

Completion of the Human Genome Project and advances in microarray and mass spectrometric technologies have enabled scientists to combine high-throughput genomic, transcriptomic and proteomic assays with defined databases to: 1) perform systematic genomic searches to identify genes differentially expressed in different tissues; 2) screen for novel markers; and 3) fingerprint distinct subclasses of tumours. High-throughput profiling of gene expression via oligonucleotide or cDNA microarrays enables researchers to characterize global transcriptional patterns in cancer cells. This systematic approach to discovery of genomic fingerprints based on unique gene expression profiles can identify patterns of molecules predictive of prognosis and response to chemotherapy. After refinement and validation in independent cohorts, these molecules can be developed into assays that accurately predict prognosis and responses to chemotherapeutic agents, thus further contributing to the development of "personalized medicine" of different combination therapies for bladder cancer patients.

As to classification and prognosis, multiple studies in recent years have demonstrated that gene expression signatures can distinguish muscle-invasive from non-invasive UC. ${ }^{44,45}$ Sanchez-Carbayo and colleagues profiled 72 muscleinvasive patients using oligonucleotide arrays to identify genetic signatures characteristic of aggressive clinical behaviour. ${ }^{45}$ The authors developed a signature consisting of 100 known genes with $90 \%$ accuracy of prediction of overall survival of muscle-invasive tumour specimens in crossvalidation studies ${ }^{45}$ Immunohistochemical validation studies demonstrated that one of the genes from the signature, synuclein, was associated with tumour stage and outcome in a tissue microarray of 294 patients. Finally, on the subject of independent validation of signatures from their prior microarray studies, Dyrskjot and colleagues recently reported an international multicentre validation study demonstrating the efficacy of their reported signatures of progression of superficial bladder tumours to muscle invasion. ${ }^{44,46,47}$
In a small study that investigated prediction of chemotherapeutic sensitivity for bladder cancer, Takata and colleagues profiled tumour response to neoadjuvant MVAC therapy using CDNA microarrays. Fourteen tumours were used to develop a signature of 14 predictive genes. ${ }^{48}$ To further validate the clinical significance of the gene expression profile system, this group recently applied it to additional cases of bladder cancer patients and found the scoring system accurately predicted clinical response. ${ }^{49}$ In addition, Als and colleagues carried out expression profiling using tumour specimens from 30 patients to develop a signature of 55 genes that highly correlated to survival time following administration of MVAC or GC. Two of these products, emmprin and survivin, were evaluated using immunohistochemistry and were found to be independent prognostic factors for response and survival after cisplatin-containing chemotherapy in patients with advanced bladder cancer. ${ }^{50}$

These key findings suggest that important histological and clinicopathological differences among tumour types may be simplified by descriptions of molecules in the tumours. To further develop "personalized selection" of different combination therapies, Havaleshko and colleagues recently developed predictive gene expression signatures for sensitivity of bladder cell lines to cisplatin, paclitaxel and gemcitabine. When these signatures were used to predict cellular sensitivities to clinically relevant, two drug combinations, they were found to predict combination sensitivities correctly in $80 \%$ of the cells, thereby laying the groundwork for prospective validation of such signatures in patients. ${ }^{51}$ Additionally, we have developed an algorithm to more generally extrapolate and predict drug sensitivity from one type of cancer to another. ${ }^{52}$ Coexpression extrapolation (COXEN) uses specialized molecular signatures to extrapolate the drug sensitivity signature of one set of tumour cells to predict drug sensitivity in another set based on gene expression data. Specifically, COXEN was used to extrapolate drug activities from the National Cancer Institute ( $\mathrm{NCI})-60$ cell line pane ${ }^{53}$ to predict drug sensitivity of bladder cancer cell lines and, of significance, from $\mathrm{NCl}-60$ cell lines to clinical responses of cancer patients treated with commonly used chemotherapy regimens. ${ }^{52}$ Of greater significance and based on the $\mathrm{NCl}-60$ cell line screen, COXEN was able to discover in silico a novel compound with high activity against human bladder cancer. These approaches highlight how new bioinformatic strategies allow extrapolation between cell lines in vitro and in patient tumours and may enable a personalized a priori selection of therapies based on molecular profiling. The objectives of current prospective studies are to determine whether COXEN can be effectively used for patient selection and drug discovery. 


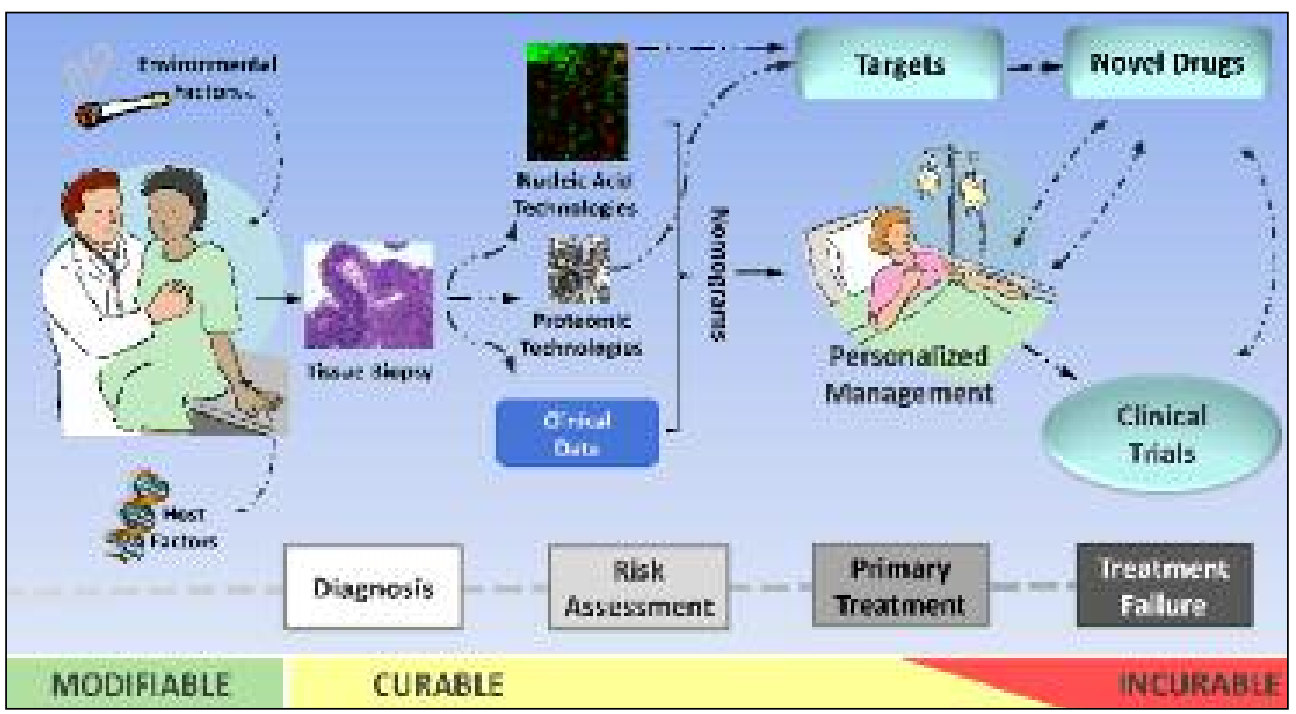

Fig. 1. Pathways to Personalized Cancer Management. Molecular profiling and highthroughput genomic, transcriptomic and proteomic assays enhance the predictive ability of standard clinicopathologic information and offer systematic approaches to identify biomarkers expressed in different tissues, to screen for novel markers and to identify active targets for drug discovery and tailored therapeutics.

\section{Conclusion}

Understanding the molecular basis of tumorigenesis and progression can lead to both identification of biomarkers and targets for therapy. Advances in high-throughput technology have demonstrated an ability to differentiate and subclassify muscle-invasive bladder cancer and potentially predict tumour response to chemotherapy. Future nomograms that incorporate pathologic and molecular information may assist physicians in accurately predicting risk of disease recurrence after definitive local therapy and result in neoadjuvant or adjuvant treatment strategies in patients with advanced disease (Fig. 1). Therefore, the choice of a combination of prognostic and therapy selection markers may be an optimal solution. For example, in the case of breast cancer, assays based on validated gene expression studies have become commercially available and proven useful in patient management. ${ }^{54}$ Hence, future research in bladder cancer should focus on validating prognostic and therapeutic selection strategies in prospective clinical trials that will contribute to the development of "personalized selection" of different combination therapies for bladder cancer patients.

From the ${ }^{*}$ Department of Urology; ${ }^{\dagger}$ Department of Molecular Physiology; ${ }^{\ddagger}$ Paul Mellon Urologic Cancer Institute, University of Virginia, Charlottesville, VA

Competing interests: None declared.

This paper has been peer-reviewed.

\section{References}

1. Parkin DM, Bray F, Ferlay J, et al. Global cancer statistics, 2002. CA Cancer I Clin 2005;55:74-108.

2. Jemal A, Siegel R, Ward E, et al. Cancer statistics, 2008. CA Cancer J Clin 2008:58:71-96.

3. Spruck $\mathrm{CH}$ 3rd, Ohneseit PF, Gonzalez-Zulueta $M$, et al. Two molecular pathways to transitional cell carcinoma of the bladder. Cancer Res 1994;54:784-8.

4. Wu XR. Urothelial tumorigenesis: a tale of divergent pathways. Nat Rev Cancer 2005;5:713-25.

5. Holmang S, Hedelin H, Anderstrom C, et al. The relationship among multiple recurrences, progression and prognosis of patients with stages Ta and $\mathrm{Tl}$ transitional cell cancer of the bladder followed for at least 20 years. J Urol 1995; 153:1823-6; discussion 1826-7.

6. Prout GR, Marshall VF. The prognosis with untreated bladder tumors. Cancer 1956;9:551-8.

7. Stein JP, Lieskovsky $G$, Cote R, et al. Radical cystectomy in the treatment of invasive bladder cancer: long-term results in 1,054 patients. J Clin Oncol 2001;19:666-75.

8. Grossman HB, Natale RB, Tangen CM, et al. Neoadjuvant chemotherapy plus cystectomy compared with cystectomy alone for locally advanced bladder cancer. N Engl J Med 2003;349:859-66.

9. Sternberg CN, de Mulder P, Schornagel JH, et al. Seven year update of an EORTC phase III trial of highdose intensity M-VAC chemotherapy and G-CSF versus classic M-VAC in advanced urothelial tract tumours. Eur J Cancer 2006;42:50-4.

10. von der Maase H, Sengelov L, Roberts JT, et al. Long-term survival results of a randomized trial comparing gemcitabine plus cisplatin, with methotrexate, vinblastine, doxorubicin, plus cisplatin in patients with bladder cancer. J Clin Oncol 2005;23:4602-8.

11. Saxman SB, Propert KJ, Einhorn LH, et al. Long-term follow-up of a phase III intergroup study of cisplatin alone or in combination with methotrexate, vinblastine, and doxorubicin in patients with metastatic urothelial carcinoma: a cooperative group study. J Clin Oncol 1997;15:2564-9.

12. Ennis RD, Petrylak DP, Singh P, et al. The effect of cystectomy, and perioperative methotrexate, vinblastine, doxorubicin and cisplatin chemotherapy on the risk and pattern of relapse in patients with muscle invasive bladder cancer. J Urol 2000;163:1413-8.

13. Slaton JW, Swanson DA, Grossman HB, et al. A stage specific approach to tumor surveillance after radical cystectomy for transitional cell carcinoma of the bladder. J Urol 1999;162(3Pt1):710-4.

14. Bassi P, Ferrante GD, Piazza N, et al. Prognostic factors of outcome after radical cystectomy for bladder cancer: a retrospective study of a homogeneous patient cohort. J Urol 1999;161:1494-7.

15. Lotan Y, Gupta A, Shariat SF, et al. Lymphovascular invasion is independently associated with overall survival, cause-specific survival, and local and distant recurrence in patients with negative lymph nodes at radical cystectomy. J Clin Oncol 2005;23:6533-9.

16. Frazier HA, Robertson JE, Dodge RK, et al. The value of pathologic factors in predicting cancer-specific survival among patients treated with radical cystectomy for transitional cell carcinoma of the bladder and prostate. Cancer 1993;71:3993-4001.

17. Bochner BH, Kattan MW, Vora KC. Postoperative nomogram predicting risk of recurrence after radical cystectomy for bladder cancer. J Clin Oncol 2006;24:3967-72.

18. Bajorin DF, Dodd PM, Mazumdar M, et al. Long-term survival in metastatic transitional-cell carcinoma and prognostic factors predicting outcome of therapy. J Clin Oncol 1999;17:3173-81. 
Ehdaie et al.

19. Esrig D, Elmajian D, Groshen $\mathrm{S}$, et al. Accumulation of nuclear p53 and tumor progression in bladder cancer. N Engl J Med 1994;331:1259-64.

20. Esrig D, Spruck CH 3rd, Nichols PW, et al. p53 nuclear protein accumulation correlates with mutations in the p53 gene, tumor grade, and stage in bladder cancer. Am J Pathol 1993;143:1389-97.

21. Sarkis AS, Dalbagni G, Cordon-Cardo C, et al. Nuclear overexpression of $p 53$ protein in transitional cell bladder carcinoma: a marker for disease progression. J Natl Cancer Inst 1993;85:53-9.

22. Shariat SF, Tokunaga H, Zhou J, et al. p53, p21, pRB, and p16 expression predict clinical outcome in cystectomy with bladder cancer. J Clin Oncol 2004;22:1014-24.

23. Tokunaga $\mathrm{H}$, Shariat SF, Green AE, et al. Correlation of immunohistochemical molecular staging of bladder biopsies and radical cystectomy specimens. Int I Radiat Oncol Biol Phys 2001;51:16-22.

24. Chatterjee SJ, Datar R, Youssefzadeh D, et al. Combined effects of p53, p21, and pRb expression in the progression of bladder transitional cell carcinoma. J Clin Oncol 2004;22:1007-13.

25. Cote RJ, Dunn MD, Chatteriee SJ, et al. Elevated and absent pRb expression is associated with bladder cancer progression and has cooperative effects with p53. Cancer Res 1998;58:1090-4.

26. Stein JP, Ginsberg DA, Grossfeld GD, et al. Effect of p21WAF1/CIP1 expression on tumor progression in bladder cancer. J Natt Cancer Inst 1998;90:1072-9.

27. Shariat SF, Ashfaq R, Sagalowsky Al, et al. Correlation of cyclin D1 and El expression with bladder cancer presence, invasion, progression, and metastasis. Hum Pathol 2006:37:1568-76.

28. Shariat SF, Ashfaq R, Sagalowsky Al, et al. Predictive value of cell cycle biomarkers in nonmuscle invasive bladder transitional cell carcinoma. J Urol 2007;177:481-7; discussion 487.

29. Karam JA, Lotan Y, Karakiewicz Pl, et al. Use of combined apoptosis biomarkers for prediction of bladder cancer recurrence and mortality after radical cystectomy. Lancet Oncol 2007;8:128-36.

30. Shariat SF, Karakiewicz PI, Ashfaq R, et al. Multiple biomarkers improve prediction of bladder cancer recurrence and mortality in patients undergoing cystectomy. Cancer 2008;112:315-25.

31. Vogelstein B, Lane D, Levine AJ. Surfing the p53 network. Nature 2000;408:307-10.

32. Malats N, Bustos A, Nascimento CM, et al. P53 as a prognostic marker for bladder cancer: a metaanalysis and review. Lancet Oncol 2005;6:678-86.

33. Mitra AP, Birkhahn M, Cote RJ. p53 and retinoblastoma pathways in bladder cancer. World I Urol 2007;25:563-71.

34. McKnight JJ, Gray SB, O'Kane HF, et al. Apoptosis and chemotherapy for bladder cancer. J Urol 2005; 173:683-90.

35. Kong $\mathrm{G}$, Shin $\mathrm{KY}$, Oh YH, et al. Bcl-2 and p53 expressions in invasive bladder cancers. Acta Oncol 1998:37:715-20

36. Smith SD, Wheeler MA, Plescia J, et al. Urine detection of survivin and diagnosis of bladder cancer. JAMA 2001;285:324-8.

37. Shariat SF, Ashfaq R, Karakiewicz PI, et al. Survivin expression is associated with bladder cancer presence, stage, progression, and mortality. Cancer 2007;109:1106-13.

38. Jebar AH, Hurst CD, Tomlinson DC, et al. Jebar AH, et al. FGFR3 and Ras gene mutations are mutually exclusive genetic events in urothelial cell carcinoma. Oncogene 2005;24:5218-25.
39. Wulfing C, Machiels J, Richel D, et al. A single arm, multicenter, open label, phase II study of lapatinib as $2 \mathrm{~L}$ treatment of pts with locally advanced/metastatic transitional cell carcinoma of the urothelial tract. Proc Am Soc Clin Oncol 2005;23(16S):401s.

40. Theodorescu D, Sapinoso LM, Conaway MR, et al. Reduced expression of metastasis suppressor RhoGDI2 is associated with decreased survival for patients with bladder cancer. Clin Cancer Res 2004;10:3800-6.

41. Titus $B$, Frierson $\mathrm{HF} \mathrm{Jr}$, Conaway $M$, et al. Endothelin axis is a target of the lung metastasis suppressor gene RhoGDI2. Cancer Res 2005;65:7320-7.

42. Smith SC, Oxford G, Wu Z, et al. The Metastasis-Associated Gene CD24 is Regulated by Ral GTPase and Is a Mediator of Cell Proliferation and Survival in Human Cancer. Cancer Res 2006;66:1917-22.

43. Smith S, Overdevest J, McRoberts K, et al. Monoclonal antibody therapy targeting CD24+ bladder cancer cells in vivo. AACR Meeting Abstracts 2008 2008;4593.

44. Dyrskiøt $L$, Thykjaer $T$, Kruhoffer $M$, et al. Identifying distinct classes of bladder carcinoma using microarrays. Nat Genet 2003;33:90-6.

45. Sanchez-Carbayo M, Socci ND, Lozano J, et al. Defining molecular profiles of poor outcome in patients with invasive bladder cancer using oligonucleotide microarrays. J Clin Oncol 2006;24:778-89.

46. Dyrskiøt L, Zieger K, Kruhøffer $M$, et al. A molecular signature in superficial bladder carcinoma predicts clinical outcome. Clin Cancer Res 2005;11:4029-36.

47. Dyrskiøt L, Zieger K, Real FX, et al. Gene expression signatures predict outcome in non-muscle-invasive bladder carcinoma: a multicenter validation study. Clin Cancer Res 2007;13:3545-51.

48. Takata R, Katagiri $T$, Kanehira $M$, et al. Predicting response to methotrexate, vinblastine, doxorubicin, and cisplatin neoadjuvant chemotherapy for bladder cancers through genome-wide gene expression profiling. Clin Cancer Res 2005;11:2625-36.

49. Takata R, Katagiri T, Kanehira M, et al. Validation study of the prediction system for clinical response of M-VAC neoadiuvant chemotherapy. Cancer Sci 2007;98:113-7.

50. Als $A B$, Dyrskiøt $L$, von der Maase $H$, et al. Emmprin and survivin predict response and survival following cisplatin-containing chemotherapy in patients with advanced bladder cancer. Clin Cancer Res 2007;13(15Pt1):4407-14

51. Havaleshko DM, Cho $\mathrm{H}$, Conaway $M$, et al. Prediction of drug combination chemosensitivity in human bladder cancer. Mol Cancer Ther 2007;6:578-86.

52. Lee JK, Havaleshko DM, Cho H, et al. A strategy for predicting the chemosensitivity of human cancers and its application to drug discovery. Proc Natl Acad Sci U S A 2007;104:13086-91.

53. Shoemaker RH. The NCI60 human tumour cell line anticancer drug screen. Nat Rev Cancer 2006;6:813-23.

54. Puszztai L. Current status of prognostic profiling in breast cancer. Oncologist 2008;13:350-60.

Correspondence: Dr. Dan Theodorescu, University of Virginia, Charlottesville, VA 22908; fax: 434-243-6878; d+9d@virginio.edu 